ORIGINAL ARTICLE

\title{
Evaluation of blunt abdominal trauma: current practice in Taiwan
}

\author{
C-F Chong, T-L Wang, H Chang
}

Emerg Med J 2005;22:113-115. doi: 10.1136/emj.2003.007328

See end of article for authors' affiliations

.....................

Correspondence to:

Dr C-F Chong, Emergency

Department, Shin Kong

Wu Ho-Su Memorial

Hospital, 95 Wen-Chang

Road, Shih Lin District,

Taipei City, Taiwan 111;

jackchong@tmu.edu.tw

Accepted for publication 9 June 2003
Objective: To gain an overview of the current practice of different major institutions in Taiwan in the evaluation of abdominal injuries. A further comparison was made between general surgeons and emergency physicians in this aspect.

Method: A telephone survey was conducted of all emergency departments of 58 major institutions (14 medical centres, 44 district hospitals) that are capable of providing definitive care for trauma victims in Taiwan in June 2002. Respondents were asked to select the diagnostic modality of choice in the evaluation of a haemodynamically abnormal blunt trauma victim with suspected intra-abdominal injuries. In the same study period, this particular telephone scenario was also used to survey 109 individual doctors (45 emergency physicians, 64 general surgeons).

Results: Most respondents preferred ultrasound (also known as focused assessment with sonography for trauma or "FAST") instead of diagnostic peritoneal lavage (DPL) because DPL is invasive and most doctors in Taiwan have limited experience in performing DPL or interpreting the results.

Conclusions: It seems reasonable to devote greater resources for emergency departments to incorporate a FAST based algorithm into their initial management of trauma victims, and to improve training in its use. It is also suggested that future ATLS teaching in Taiwan should include didactic material on FAST.
$\mathrm{T}$ he abdomen of trauma victims is routinely evaluated with physical examination. However, physical examination may be misleading in patients under the influence of alcohol or illicit drugs. It is also unreliable in patients with associated injuries to the head or spinal cord. ${ }^{12}$ Diagnostic peritoneal lavage (DPL), ultrasound (FAST), and computed tomography (CT) are typical tests used for abdominal evaluation in trauma. FAST has been shown to be as accurate as DPL and CT in the detection of haemoperitoneum after abdominal trauma. ${ }^{3-5}$ Current guidelines as taught in the Advanced Trauma Life Support (ATLS) courses throughout the world suggest that DPL should be performed in trauma victims with suspected intra-abdominal injuries. Techniques and interpretation of findings of DPL are also taught in the ATLS surgical skills practicum. ${ }^{6}$

Since its introduction in 1996, the "ATLS Provider" training programme has benefited more than 600 doctors in Taiwan. Most of them were surgeons and emergency physicians. $^{7}$ Although most surgeons and emergency physicians know DPL well, it is not the primary initial diagnostic modality for the evaluation of blunt trauma victims with suspected intra-abdominal injuries in Taiwan. Ultrasound, on the other hand, has become a standard of care in most emergency departments (EDs). ${ }^{3}$ When used for trauma evaluation, it is also known as focused assessment with sonography for trauma (FAST).

The main objective of this study was to assess the current practice of different major institutions (medical centres and district hospitals) in Taiwan in the initial evaluation of abdominal injuries. Another objective is to discover if there is a difference between general surgeons and emergency physicians in this aspect.

\section{METHODS}

In June 2002, we conducted a telephone survey of all EDs of 58 major institutions (14 medical centres and 44 district hospitals) that are capable of providing definitive care for trauma victims in Taiwan. A single researcher was assigned to make the telephone calls. The respondent was either an attending physician (when available) or a senior residence on duty in the ED. Questions asked include: (1) the first choice diagnostic modality (choose among DPL, FAST, and CT) to evaluate the abdomen of a blunt trauma victim with a systolic blood pressure of $80 \mathrm{~mm} \mathrm{Hg}$ and a pulse of $130 \mathrm{bpm}$ on presentation at the ED; and (2) whether or not he or she is an ATLS provider. Non-DPL respondents were further asked for their reasons. Any additional comments were noted. A repeat phone call was made 24 hours later if initial contact was unsuccessful.

In addition, a list of 194 board certified emergency physicians (1999 to 2001) and 330 board certified general surgeons (1999 to 2001) was identified. Every third case in the list was randomly selected to derive our study sample that finally consists of 58 emergency physicians (EP group) and 99 general surgeons (GS group). Another telephone survey of this study population was conducted. The same researcher was assigned to make the telephone calls asking the same questions as mentioned before. Non-DPL respondents were further asked for their reasons.

All collected variables were categorical and were compared using the $\chi^{2}$ test. Analyses were performed on SPSS 10.0 for Windows (SPSS) with statistical significance set at $\mathrm{p}<0.05$ (two tailed).

\section{RESULTS}

In the telephone survey of institutions, we received responses from all of the major institutions, including 14 medical centres (MC) and 44 district hospitals (DH). As table 1 shows, $78.6 \%$ of $\mathrm{MC}$ respondents and $81.8 \%$ of $\mathrm{DH}$ respondents preferred FAST. Only $7.1 \%$ of MC respondents and $6.8 \%$ of DH respondents preferred DPL. The remaining $14.3 \%$

Abbreviations: FAST, focused assessment with sonography for trauma; $D P L$, diagnostic peritoneal lavage; $C T$, computed tomography; $E D$, emergency department; GS, general surgeon; EP, emergency physician; $M C$, medical centre; DH, district hospital 
Table 1 Diagnostic preferences

\begin{tabular}{|c|c|c|c|c|c|c|}
\hline & \multicolumn{3}{|c|}{ Institutions, n (\%) } & \multicolumn{3}{|c|}{ Participants, n (\%) } \\
\hline & $M C$ & DH & p Value & EP & GS & p Value \\
\hline FAST & $11(78.6)$ & $36(81.8)$ & 0.81 & 40 (88.9) & 47 (73.4) & 0.21 \\
\hline DPL & $1(7.1)$ & $3(6.8)$ & 1.00 & $2(4.4)$ & $4(6.3)$ & 0.53 \\
\hline $\mathrm{CT}$ & $2(14.3)$ & $5(11.4)$ & 0.55 & $3(6.7)$ & $13(20.3)$ & 0.01 \\
\hline Total & $14(100)$ & $44(100)$ & & $45(100)$ & 64 (100) & \\
\hline
\end{tabular}

MC, medical centre; DH, district hospital; EP, emergency physician; GS, general surgeon; FAST, focused assessment with sonography for trauma; DPL, diagnostic peritoneal lavage; CT, computed tomography.

of MC respondents and $11.4 \% \mathrm{DH}$ respondents preferred CT in the assessment of the haemodynamically unstable patient. There was no significant difference between the two groups. Table 2 shows that nearly all $(>90 \%)$ respondents selected not to perform DPL because they believed that DPL carries more risks. The DH group consisted of more respondents who had limited experience in performing and interpreting DPL than the MC group (70.5\% compared with $35.7 \%, p=0.02$ ). A significantly higher percentage of ATLS provider was found in the MC group than the DH group $(85.7 \%$ compared with $59.1 \%, \mathrm{p}=0.03$ ).

In the telephone survey of individual doctors, 45 (77.6\%) of the 58 randomly selected EPs and $64(64.7 \%)$ of the 99 randomly selected GSs responded to our questions. As table 1 shows, $88.9 \%$ of EP respondents and $73.4 \%$ of GS respondents preferred FAST. Only $4.4 \%$ of EP respondents and $6.3 \%$ of GS respondents preferred DPL. There were significantly more GS respondents than EP respondents (20.3\% compared with $6.7 \%, \mathrm{p}=0.01$ ) that preferred CT examination in the scenario. Table 2 showed that nearly all EP and GS respondents selected not to perform DPL because of its invasiveness although most of them had experience in performing and interpreting DPL. A significantly higher percentage of ATLS provider was found in the EP group than the GS group (53.3\% compared with $12.5 \%, \mathrm{p}<0.01$ ).

\section{DISCUSSION}

Unrecognised abdominal injury is a frequent cause of preventable death after trauma. Clinical signs may be subtle or masked by other injuries or intoxicants. ${ }^{4}$ Successful care of patients with blunt abdominal trauma depends on accurate and rapid identification of those patients requiring operative intervention for intra-abdominal injuries. However, the most effective method of evaluation remains controversial. Current diagnostic modalities include DPL, CT, and FAST. ${ }^{589}$

DPL is a rapidly performed, invasive procedure that provides useful information in the evaluation of blunt trauma victims ${ }^{10}$ and is considered $98 \%$ sensitive for intraperitoneal bleeding. ${ }^{11}$ It is recommended in the ATLS guidelines that DPL should be performed in haemodynamically abnormal patients with multiple blunt injuries, when associated with altered sensorium, injury to adjacent structures, equivocal physical findings, or when a prolonged loss of contact with patient is anticipated (lengthy radiological studies, general anaesthesia for extra-abdominal injuries, etc). ${ }^{6}{ }^{12}$ The advantages of DPL are simplicity, accuracy, and low complication rates. ${ }^{11} 12$ DPL is considered oversensitive. Only a small volume of free intraperitoneal blood can lead to a positive result. False positive results can occur from a retroperitoneal leak, which cannot detect contained solid organ injury but can confound later computed tomograms by introducing both air and fluid into the peritoneal cavity. Furthermore, there is justified concern regarding familiarity with the lavage techniques, collection, processing, quantification, and interpretation of results in many institutions where DPL is only occasionally seen. ${ }^{10}{ }^{13}{ }^{14}$ This, together with a high incidence of non-therapeutic celiotomy and a shift to a more conservative approach to the treatment of solid organ injury, has resulted in DPL being redefined in the diagnosis of blunt abdominal trauma.

Ultrasound has an expanding role in the assessment of blunt abdominal injuries. It is a valuable tool for the diagnosis of haemoperitoneum requiring celiotomy. ${ }^{15}$ The advantages of ultrasound, in comparison with CT and DPL, are rapidity of the examination to detect free fluid, portability, and lack of need for patient transport. Ultrasound can be performed during trauma resuscitation, on patients with previous celiotomies, on patients with clotting disorders, and on pregnant patients. Limitations of ultrasound include technical difficulties in obese patients and in patients with ascites, subcutaneous emphysema, or hollow organ injury. Although ultrasound cannot replace CT or DPL, it is a rapid screening method for detection of haemoperitoneum and for guidance of further diagnostic studies. ${ }^{16}$

Our survey showed that most major institutions and most individual doctors in Taiwan prefer FAST as the initial test of choice when encountering a haemodynamically abnormal trauma victim with suspected abdominal injuries. This is true in all study groups (MC, DH, EP, and GS), although the degree of exposure to ATLS training among these groups is quite different (more ATLS providers found in the MC and EP groups). In Taiwan, ultrasound machines are commonly available and FAST is considered as the initial diagnostic modality of choice to exclude haemoperitoneum. Most trauma doctors in Taiwan will decide to carry out DPL only when ultrasound is unavailable and the patient is in shock.

In Taiwan, CT scanners are available in all institutions that manage traumatised patients. CT takes longer, costs more, and should not be done in the unstable patients, but it gives more definitive information about the abdominal organs and retroperitoneum. ${ }^{17} \mathrm{CT}$ is the diagnostic modality of choice for non-operative management of solid visceral injuries. The literature suggests that CT carries comparable sensitivity and

Table 2 Reasons for not performing DPL

\begin{tabular}{|c|c|c|c|c|c|c|}
\hline & \multicolumn{3}{|c|}{ Institutions, n (\%) } & \multicolumn{3}{|c|}{ Participants, n (\%) } \\
\hline & $M C$ & $\mathrm{DH}$ & p Value & EP & GS & p Value \\
\hline Inexperience & 5 (35.7) & $31(70.5)$ & 0.02 & $43(95.4)$ & $54(84.4)$ & 0.37 \\
\hline Invasiveness & $13(92.9)$ & 43 (97.7) & 0.66 & $45(100)$ & $60(93.8)$ & 0.61 \\
\hline
\end{tabular}


superior specificity as compared with DPL and it is contraindicated in haemodynamically abnormal patients. ${ }^{18}$ In our study, we found a minority of respondents (more surgeons than emergency physicians) decided to send haemodynamically abnormal patients to the radiology department for CT. They claimed that all patients are well monitored, the CT scanner is in the ED or immediately adjacent to the trauma resuscitation room, and rapid examination is anticipated with newer generation CT scanners.

Some authors recommended that the next edition of the ATLS manual should include didactic material on ultrasound. ${ }^{14}{ }^{19}$ However, the inclusion of practical ultrasound instruction in future ATLS courses will not be universally applicable because of limited availability of ultrasound equipment and lack of competent instructors. ${ }^{19}$ This seems not to be a problem in Taiwan as ultrasound equipment is commonly available and FAST has long been part of the standard ED residence training programme.

There are several limitations to this study. Firstly, only one telephone call is made for each hospital ED. A different respondent from the same ED may have given a different answer. Secondly, only large scale institutions are included in the study. Local hospitals especially those in the rural areas are not included. Thirdly, the representativeness of our database is limited to Taiwan only and may not applicable to other localities.

Although it is well known that DPL is accurate, rapid, safe, and avoids the disruption of patient care, it is not the primary test for the evaluation of trauma victims with suspected intra-abdominal injuries in Taiwan, as shown in this study. Recognising its value as a rapid, accurate, non-invasive diagnostic modality, most EDs in Taiwan has included ultrasound in their algorithm for the assessment of trauma victims. It seems reasonable to devote greater resources for EDs to incorporate a FAST based algorithm into their initial management of trauma victims, and to improve training in its use. We also suggested that future ATLS teaching in Taiwan should include didactic material on FAST.

\section{CONTRIBUTORS}

CF Chong collected, organised, and interpreted the data and wrote the paper. TL Wang and H Chang supervised the study.

\section{Authors' affiliations}

C-F Chong, T-L Wang, H Chang, Emergency Department, Shin Kong Wu Ho-Su Memorial Hospital, Taipei, Taiwan, ROC
Funding: none.

Conflicts of interest: none declared.

\section{REFERENCES}

1 Bivins BA, Sachatello CR, Daugherty ME, et al. Diagnostic peritoneal lavage is superior to clinical evaluation in blunt abdominal trauma. Am Surg 1978;44:637-41

2 Rodriguez A, DuPriest RW, Shatney $\mathrm{CH}$. Recognition of intra-abdominal injury in blunt traum victims: a prospective study comparing physical examination with peritoneal lavage. Am Surg 1982;48:457-9.

3 Scalea TM, Rodriguez A, Chiu WC, et al. Focused assessment with sonography for trauma (FAST): results from an international consensus conference. J Trauma 1999:46:466-72.

4 Rozycki GS, Shackford SR. Ultrasound: What every trauma surgeon should know. J Trauma 1996;40:1-4

5 Liv M, Lee C, $P^{\prime}$ eng F. Prospective comparison of diagnostic peritoneal lavage, computed tomographic scanning, and ultrasonography for the diagnosis of blunt abdominal trauma. J Trauma 1993;35:267-70.

6 American College of Surgeons Committee on Trauma. Advanced Trauma Life Support for doctors. Student course manual. 6th ed. Chicago: American College of Surgeons, 1997.

7 Chen SC, Tsai MC, Liu HD, et al. Advanced Trauma Life Support provider course in Taiwan. J Emerg Crit Care Med 2001;12:111.

8 Brown CK, Dunn KA, Wilson K. Diagnostic evaluation of patients with blunt abdominal trauma: a decision analysis. Acad Emerg 2000;7:385-96.

9 Nordenholz KE, Rubin MA, Gularte GG, et al. Ultrasound in the evaluation and management of blunt abdominal trauma. Ann Emerg Med 1997;29:357-66.

10 Chiquito PE. Blunt abdominal injuries. Diagnostic peritoneal lavage, ultrasonography and computed tomography scanning. Injury 1996;27:117-24.

11 Nagy KK, Roberts RR, Joseph KT, et al. Experience with over 2500 diagnostic peritoneal lavages. Injury 2000;31:479-82.

12 Hawkins ML, Bailey RL, Carraway RP. Is diagnostic peritoneal lavage for blunt trauma obsolete? Am Surg 1990;56:96-9.

13 Velmahos GC, Demetriades D, Stewart $M$, et al. Open versus closed diagnostic peritoneal lavage: a comparison on safety, rapidity, efficacy. $J$ R Coll Surg Edinb 1998;43:235-8.

14 Maxwell-Armstrong C, Brooks A, Field $M$, et al. Diagnostic peritoneal lavage analysis: should trauma guidelines be revised? Emerg Med J 2002;19:524-5.

15 McKenney KL. Ultrasound of blunt abdominal trauma. Radiol Clin North Am 1999;37:879-93.

16 Bain IM, Kirby RM, Tiwari P, et al. Survey of abdominal ultrasound and diagnostic peritoneal lavage for suspected intra-abdominal injury following blunt trauma. Injury 1998;29:65-7.

17 Meyer DM, Thal ER, Weigelt JA, et al. Evaluation of computed tomography and diagnostic peritoneal lavage in blunt abdominal trauma. J Trauma 1989;29: $1168-70$

18 Bell C, Coleridge ST. A comparison of diagnostic peritoneal lavage and computed tomography (CT scan) in evaluation of the haemodynamically stable patient with blunt abdominal trauma. J Emerg 1992; 10:275-80.

19 Han DC, Rozycki GS, Schmidt JA, et al. Ultrasound training during ATLS: an early start for surgical interns. J Trauma 1996;41:208-13. 